\title{
Triviality and stability in effective theories
}

\author{
José Wudka* \\ Department of Physics, University of California, Riverside CA 92521-0413, USA \\ E-mail: 'jose.wudkāoucr.edū'
}

\section{Bohdan Grzadkowski}

Institute of Theoretical Physics, Warsaw University, Hoża 69, PL-00-681 Warsaw, POLAND

E-mail: bohdan. grzadkowski@fuw.edu.pil

\begin{abstract}
In this talk we consider the modifications induced by heavy physics on the triviality and vacuum stability bounds on the Higgs-boson mass. We parameterize the heavy interactions using an effective Lagrangian and find that the triviality bound is essentially unaffected for weakly-coupled heavy physics. In contrast there are significant modifications in the stability bound that for a light Higgs boson require a scale of new physics of the order of a few TeV.
\end{abstract}

Introduction The structure of the gauge symmetry breaking sector of the standard model (SM) remains one of the most interesting unknowns of this theory. Assuming a scalar sector with a single doublet, LEP obtained a bound on the Higgs-boson mass of [i]1, $m_{H}>113.2 \mathrm{GeV}$ (mainly using the reaction $e^{+} e^{-} \rightarrow Z H$ ). For this particular scalar sector, existing precision measurements can be used to provide the upper limit $m_{H}<220 \mathrm{GeV}$ [i2].

These results, despite their being very model dependent, suggest the presence of light

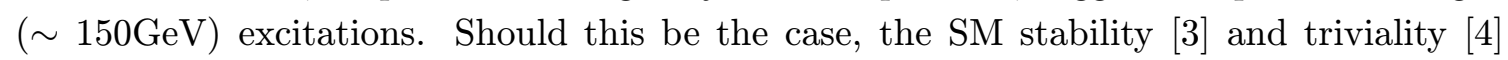
bounds strongly favor the appearance of new physics at scales $\lesssim 100 \mathrm{TeV}$. Most calculations of these bounds assume either a specific model, or else assume pure SM interactions up to a scale $\Lambda$ where new physics abruptly "kicks in". In this talk we review [to these results when the new physics has a characteristic scale $\Lambda$ significantly above the Fermi scale, yet it does have effects at low energies (albeit suppressed by powers of $1 / \Lambda$ ).

New physics effects Assuming $\Lambda$ is much larger than the Fermi scale allows us to parameterize the heavy physics effects below $\Lambda$ using an effective Lagrangian of the form

$$
\mathcal{L}_{\text {eff }}=\frac{1}{\Lambda^{2}} \sum_{i} \alpha_{i} \mathcal{O}_{i}+O\left(1 / \Lambda^{3}\right)
$$

\footnotetext{
${ }^{*}$ Speaker.
} 
where the $\mathcal{O}_{i}$ are gauge invariant operators constructed using the SM fields, and the $\alpha_{i}$ (calculable if the underlying theory is known) parameterize the new-physics effects.

We also assume that the underlying physics is weakly coupled, so that operators generated at tree level dominate [i] and that chiral symmetry is natural [i]

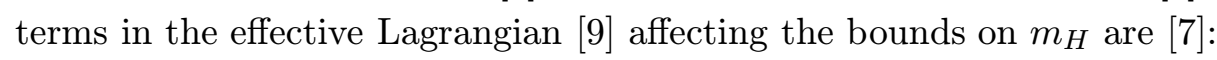

$$
\begin{aligned}
& \mathcal{O}_{\phi}=\frac{1}{3}|\phi|^{6} \quad \mathcal{O}_{\partial \phi}=\frac{1}{2}\left(\partial|\phi|^{2}\right)^{2} \quad \mathcal{O}_{\phi}^{(1)}=|\phi|^{2}|D \phi|^{2} \\
& \mathcal{O}_{\phi}^{(3)}=\left|\phi^{\dagger} D \phi\right|^{2} \quad \mathcal{O}_{t \phi}=|\phi|^{2}(\bar{q} \tilde{\phi} t+\text { h.c. }) \quad \mathcal{O}_{q t}^{(1)}=\frac{1}{2}|\bar{q} t|^{2},
\end{aligned}
$$

where $\phi$ denotes the SM scalar doublet, $q$ the left-handed top-bottom isodoublet and $t$ the right-handed top isosinglet ${ }^{1}$. We also define $\eta \equiv \lambda v^{2} / \Lambda^{2}$, where $v$ denotes the SM vacuum expectation value, $v \sim 246 \mathrm{GeV}$. The full Lagrangian we will use is then

$$
\begin{aligned}
\mathcal{L}_{\text {tree }} & =-\frac{1}{4}\left(F^{2}+B^{2}\right)+|D \phi|^{2}+i \bar{q} \not D q+i \bar{t} \not D t \\
& +f(\bar{q} \tilde{\phi} t+\text { h.c. })-\lambda\left(|\phi|^{2}-v^{2} / 2\right)^{2}+\sum_{i} \frac{\alpha_{i}}{\Lambda^{2}} \mathcal{O}_{i} .
\end{aligned}
$$

It is worth noting that in this model one can do perturbative calculations; power divergences are relevant for naturality arguments while logarithmic divergences determine the renormalization group (RG) evolution of the $\alpha_{i}$ in the usual manner [i $[\dot{i}]$.

Triviality We demand that our theory remains perturbative throughout its RG evolution: $|\lambda(\kappa)|<\lambda_{\max },\left|\alpha_{i}(\kappa)\right|<\alpha_{\max }$ for $\kappa<\Lambda$. The RG equations to one loop are

$$
\begin{aligned}
\frac{d \lambda}{d t}= & 12 \lambda^{2}-3 f^{4}+6 \lambda f^{2}-\frac{3 \lambda}{2}\left(3 g^{2}+g^{\prime 2}\right)+\frac{3}{16}\left(g^{\prime 4}+2 g^{2} g^{2}+3 g^{4}\right) \\
& -2 \eta\left[2 \alpha_{\phi}+\lambda\left(3 \alpha_{\partial \phi}+4 \bar{\alpha}+\alpha_{\phi}^{(3)}\right)\right] \\
\frac{d \eta}{d t}= & 3 \eta\left[2 \lambda+f^{2}-\frac{1}{4}\left(3 g^{2}+g^{\prime 2}\right)\right]-2 \eta^{2} \bar{\alpha} \\
\frac{d f}{d t}= & \frac{9 f^{3}}{4}-\frac{f}{2}\left(8 g_{s}^{2}+\frac{9}{4} g^{2}+\frac{17}{12} g^{\prime 2}\right)-\frac{f \eta}{2}\left(-6 \frac{\alpha_{t \phi}}{f}+\bar{\alpha}+3 \alpha_{q t}^{(1)}\right) \\
\frac{d \alpha_{\phi}}{d t}= & 9 \alpha_{\phi}\left(6 \lambda+f^{2}\right)+12 \lambda^{2}\left(9 \alpha_{\partial \phi}+6 \alpha_{\phi}^{(1)}+5 \alpha_{\phi}^{(3)}\right)+36 \alpha_{t \phi} f^{3} \\
& -\frac{9}{8}\left[2\left(3 g^{2}+g^{\prime 2}\right) \alpha_{\phi}+2 \alpha_{\phi}^{(1)} g^{4}+\left(\alpha_{\phi}^{(1)}+\alpha_{\phi}^{(3)}\right)\left(g^{2}+g^{2}\right)^{2}\right] \\
\frac{d \alpha_{\partial \phi}}{d t}= & 2 \lambda\left(6 \alpha_{\partial \phi}-3 \alpha_{\phi}^{(1)}+\bar{\alpha}\right)+6 f\left(f \alpha_{\partial \phi}-\alpha_{t \phi}\right) \\
\frac{d \alpha_{\phi}^{(1)}}{d t}= & 2 \lambda\left(\bar{\alpha}+3 \alpha_{\phi}^{(1)}\right)+6 f\left(f \alpha_{\phi}^{(1)}-\alpha_{t \phi}\right) \\
\frac{d \alpha_{\phi}^{(3)}}{d t}= & 6\left(\lambda+f^{2}\right) \alpha_{\phi}^{(3)} \\
\frac{d \alpha_{t \phi}}{d t}= & -3 f\left(f^{2}+\lambda\right) \alpha_{q t}^{(1)}+\left(\frac{15}{4} f^{2}-12 \lambda\right) \alpha_{t \phi}-\frac{f^{3}}{2}\left(\alpha_{\partial \phi}-\alpha_{\phi}^{(1)}+\bar{\alpha}\right)
\end{aligned}
$$

\footnotetext{
${ }^{1}$ The operator $\mathcal{O}_{q t}^{(1)}$ affects the stability bound only through RG mixing and its effects are small; other similar operators were not included for this reason.
} 


$$
\frac{d \alpha_{q t}^{(1)}}{d t}=(3 / 2) \alpha_{q t}^{(1)} f^{2}
$$

where $\kappa=M_{Z} \exp \left(8 \pi^{2} t\right)$ is the renormalization scale, and $\bar{\alpha}=\alpha_{\partial \phi}+2 \alpha_{\phi}^{(1)}+\alpha_{\phi}^{(3)}$. The evolution of the gauge couplings $g, g^{\prime}$ and $g_{s}$ (for the strong interactions) is unaffected by the $\alpha_{i}$ 's. These equations are solved using the following boundary conditions: $\alpha_{i}(\Lambda)=$ $O(1)$ (with various sign choices); $\langle\phi\rangle=0.246 / \sqrt{2} \mathrm{TeV}$ (at $\kappa=M_{Z}$ ) and, finally, that the $W, Z, t, H$ masses have their physical values. Sample plots of the running couplings are given in Fig.'i'.'.

Using the RG solutions and the above condition we find the triviality bounds plotted in Fig. $i_{1}^{1}$ These bounds are indistinguishable from the SM due to our requirement that the model remains weakly coupled; if this is relaxed our conclusions need not hold [i] $\left.{ }^{1} \overline{0}\right]$.
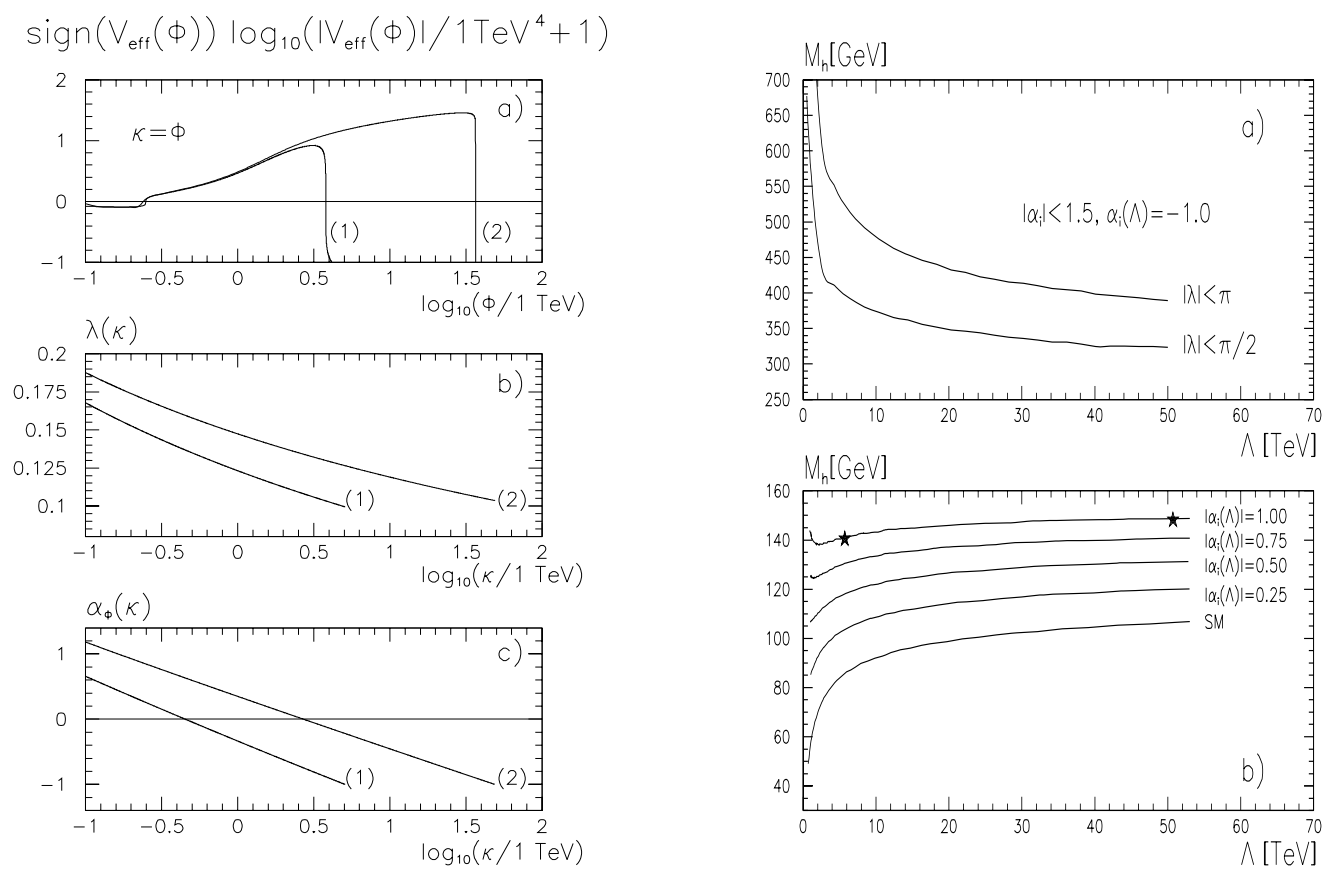

Figure 1: Left panel: (a) $V_{\text {eff }}$ at the scale $\kappa=\phi$ as a function of the field strength. The running of $\lambda$ (b) and $\alpha_{\phi}$ (c) when $\alpha_{i}(\Lambda)=-1, m_{t}=175 \mathrm{GeV}$, for $\Lambda=5.1 \mathrm{TeV}, m_{H}=140.4 \mathrm{GeV}$ (curves (1)) and $\Lambda=48.9 \mathrm{TeV}, m_{H}=148.7 \mathrm{GeV}$ (curves (2)). Right panel: Triviality (a) and stability (b) bounds on $m_{H}$ for $m_{t}=175 \mathrm{GeV}$. Stars correspond to solutions (1) and (2).

Stability We define the effective potential in terms of the zero-momentum 1PI $n$-point functions, $V_{\text {eff }}(\bar{\varphi})=-\sum_{n} \Gamma^{(n)}(0) \bar{\varphi}^{n} / n$ ! and we require that the SM vacuum is stable, that is

$$
\left.V_{\text {eff }}(\bar{\varphi}=0.75 \Lambda)\right|_{\kappa=0.75 \Lambda}=\left.V_{\text {eff }}\left(\bar{\varphi}=v_{\text {phys }} / \sqrt{2}\right)\right|_{\kappa=v_{\text {phys }} / \sqrt{2}} .
$$

The calculations were done using the explicit 1-loop expression for $V_{\text {eff }}$,

$$
V \operatorname{eff}(\bar{\varphi})=-\eta \Lambda^{2}|\phi|^{2}+\lambda|\phi|^{4}-\frac{\alpha_{\phi}}{3 \Lambda^{2}}|\phi|^{6}+\frac{1}{64 \pi^{2}} \sum_{i=0}^{5} c_{i} R_{i}^{2}\left[\ln \left(R_{i} / \kappa^{2}\right)-\nu_{i}\right]+O\left(1 / \Lambda^{4}\right),
$$


where $c_{0}=-4, c_{1}=1, c_{2,4}=3, c_{3}=6, c_{5}=-12, \nu_{0,1,2,5}=3 / 2, \nu_{3,4}=5 / 6, R_{0}=\eta \Lambda^{2}$,

$$
\begin{aligned}
& R_{1}=\lambda\left(6|\bar{\varphi}|^{2}-v^{2}\right)\left[1-\left(2 \alpha_{\partial \phi}+\alpha_{\phi}^{(1)}+\alpha_{\phi}^{(3)}\right)|\bar{\varphi}|^{2} / \Lambda^{2}\right]-5 \alpha_{\phi}|\bar{\varphi}|^{4} / \Lambda^{2} \\
& R_{2}=\lambda\left(2|\bar{\varphi}|^{2}-v^{2}\right)\left[1-\left(\alpha_{\phi}^{(1)}+\alpha_{\phi}^{(3)} / 3\right)|\bar{\varphi}|^{2} / \Lambda^{2}\right]-\alpha_{\phi}|\bar{\varphi}|^{4} / \Lambda^{2} \\
& R_{3}=\left(g^{2} / 2\right)|\bar{\varphi}|^{2}\left(1+|\bar{\varphi}|^{2} \alpha_{\phi}^{(1)} / \Lambda^{2}\right) \\
& R_{4}=\left[\left(g^{2}+g^{\prime 2}\right) / 2\right]|\bar{\varphi}|^{2}\left(1+|\bar{\varphi}|^{2}\left(\alpha_{\phi}^{(1)}+\alpha_{\phi}^{(3)}\right) / \Lambda^{2}\right) \\
& R_{5}=f|\bar{\varphi}|^{2}\left(f+2 \alpha_{t \phi}|\bar{\varphi}|^{2} / \Lambda^{2}\right),
\end{aligned}
$$

This has the same form as in the SM, but with modified $R_{i}$. Note that $V_{\text {eff }}$ is gauge dependent [i] $\left.{ }_{1}^{1}\right]$ but the effects of this gauge dependence are small since the RG-improved tree-level effective potential is gauge-invariant; this leads to a variation in the Higgs-boson mass limit of $\Delta m_{H} \lesssim 0.5 \mathrm{GeV}$ [i] $\left.\overline{2}_{2}\right]$. Using the anomalous dimension for the scalar field, $\gamma=3 f^{2} / 2-3\left(3 g^{2}+g^{\prime 2}\right) / 8-\eta \bar{\alpha} / 2$, and a careful definition of $V_{\text {eff }}(0)$ [i] $\overline{3}$ i], one can verify that $V_{\text {eff }}$ is scale invariant.

Plots of the effective potential for some representative values of the parameters and the associated stability bounds are presented in Fig is It is noteworthy that in contrast with the triviality bounds the presence of the effective operators has a significant impact on the stability bounds. For example for a Higgs-boson mass of $115 \mathrm{GeV}$,

$$
\begin{array}{ll}
\Lambda \lesssim 24 \mathrm{TeV} & \text { for }\left|\alpha_{i}\right|=0.25 \\
\Lambda \lesssim 4 \mathrm{TeV} & \text { for }\left|\alpha_{i}\right|=0.50 \\
\Lambda \lesssim 1 \mathrm{TeV} & \text { for }\left|\alpha_{i}\right|=0.60
\end{array}
$$

We also find that the main effects on the stability bound are generated by $\alpha_{\phi}, \alpha_{t \phi}$. For example, for $\alpha_{\phi}$ large and positive the potential has no minimum for fields below $0.75 \Lambda$; more precisely, there is a region in the $\alpha_{\phi}-\alpha_{t \phi}$, given in Fig. $\overline{2}_{1}^{\prime}$, where the SM vacuum is either absent or unstable for $\bar{\varphi}<0.75 \Lambda$.

Conclusions The SM triviality upper bound remains unmodified for weakly coupled heavy physics, while the stability bound increases by $\sim 50 \mathrm{GeV}$ depending on $\Lambda$ and $\alpha_{i}(\Lambda)$. For $m_{H}$ close to its lower LEP limit the constraint on $\Lambda$ could be decreased dramatically even for modest values of the $\alpha_{i}$ (different values of $\alpha_{i}$ correspond to different heavy-physics theories). These results complement the ones obtained within specific models [i] $\left.{ }_{1}^{1} \overline{4}\right]$.

Note that, strictly speaking, our expression for $V_{\text {eff }}$ is not valid at points where it

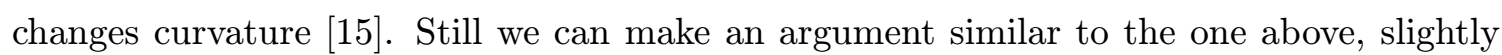
below the inflection point $|\bar{\varphi}| \sim 0.75 \Lambda$; the resulting bounds are essentially unchanged due to the precipitous drop of $V_{\text {eff }}$ beyond this point (see Fig.'1!ili).

\section{References}

[1] T. Junk [LEP Higgs Working Group] LEP Fest, Oct. 10 2000, http://lephiggs.web.cern.ch/LEPHIGGS/talks/index.html.

[2] E. Tournefier, [LEP Electroweak Working Group], 36th Rencontres De Moriond On Electroweak Interactions And Unified Theories, 2001, Les Arcs, France. 


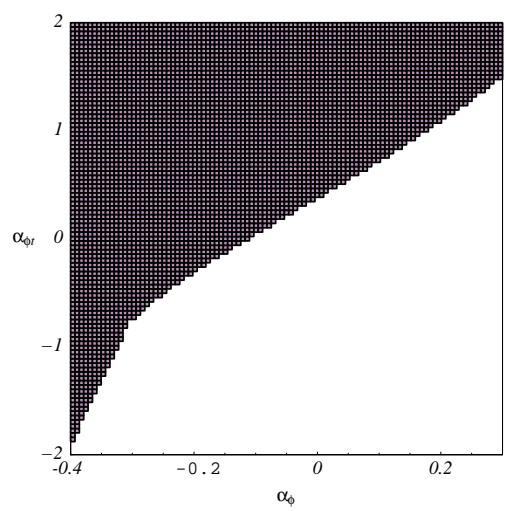

Figure 2: The unshaded region corresponds to the values of $\alpha_{\phi}(\Lambda), \alpha_{\phi t}(\Lambda)$ where the effective potential has no SM minimum for fields below $0.75 \Lambda$, for any choice of $0.5 \mathrm{TeV}<\Lambda<50 \mathrm{TeV}$.

[3] N. Cabibbo et al., Nucl. Phys. B158 (1979), 295; for a review see M. Sher, Phys. Rep. 179 (1989), 273.

[4] L. Maiani, G. Parisi, and R. Petronzio, Nucl. Phys. B136 (1979), 115; M. Lindner, Z. Phys. C31 (1986), 295.

[5] B. Grzadkowski and J. Wudka, arXiv:hep-ph/0106233.

[6] S. Weinberg, hep-th/9702027; H. Georgi, Ann. Rev. Nucl. Part. Sci. 43, 209 (1993).

[7] C. Arzt, M. B. Einhorn and J. Wudka, Nucl. Phys. B 433, 41 (1995) [arXiv:hep-ph/9405214].

[8] G. 't Hooft, Lecture given at Cargese Summer Inst., Cargese, France, Aug 26 - Sep 8, 1979, in $C^{79}$-08-26.4.

[9] W. Buchmüeller and D. Wyler, Nucl. Phys. B268 (1986), 621.

[10] M. Chanowitz, Phys. Rev. D63 (2001), 076002.

[11] W. Loinaz and R.S. Willey, Phys. Rev. D56 (1997), 7416.

[12] J. A. Casas et al., Nucl. Phys. B 436, 3 (1995) [Erratum-ibid. B 439, 466 (1995)]; M. Quiros, hep-ph/9703412.

[13] C. Ford et al., Nucl. Phys. B395 (1993), 17.

[14] J.A. Casas et al., Phys. Lett. B342 (171), 1995. A. Datta and X. Zhang, Phys. Rev. D61 (074033), 2000. S. Nie and Marc Sher, Phys. Lett. B449 (89), 1999. I. Dasgupta et al., Phys. Lett. B447 (284), 1999. J. McDonald, Phys. Lett. B413 (30), 1997. J. Velhinho et al., Phys. Lett. B322 (213), 1994 J. Sirkka and I. Vilja, Phys. Lett. B332 (141), 1994. J. Freund et al., Phys. Lett. B280 (267), 1992 B. Grzadkowski and A. Pich, Phys. Lett. B183 (71), 1987.

[15] We thank J. Kuti for this remark. 